\title{
High-pressure water injection injury
}

\author{
Christopher Scott Sampson
}

Received: 14 January 2008 / Accepted: 31 March 2008 /Published online: 17 June 2008

(C) Springer-Verlag London Ltd 2008

\section{Introduction}

Patients that present with high-pressure injection injuries most often have hand or digital involvement. Overall, highpressure injection injuries to the hand are uncommon and tend to be occupation related. The usual cause is industrial equipment, with machinery such as grease guns, spray guns and diesel engine injectors accounting for most of these injuries. Materials injected include the following reported substances: paint, paint thinner, grease, oil, hydraulic fluid, plastic, wax, water and semifluid cement.

The extent of damage from high-pressure injection injuries depends on a number of physical, chemical and biological factors, including the type, amount and velocity of injected material and the anatomical location of the injury. For any injury that results in a significant depth of penetration, the most important determinant of the extent of injury is the chemical composition of the injected material. This determines the likely tissue inflammatory response and the resulting fibrosis that develops during healing. Paint and paint thinner produce a large, early inflammatory response and result in a high percentage of amputations. In contrast, grease injuries cause a small inflammatory response and have a lower amputation rate but are associated with oleogranuloma formation, fistulas, scarring and loss of digit function. The amount of material injected into the confined space of the digits or palm determines the degree of mechanical distention and the potential for

C. S. Sampson $(\square)$

Washington University in Saint Louis,

P.O. Box 8072, 660 South Euclid,

Saint Louis, MO 63110, USA

e-mail: sampx@yahoo.com vascular compromise. The velocity of the injected material and the site of tissue penetration determine anatomical dispersion, which for upper extremity injuries may include the digit, hand and forearm [1].

The patient who seeks treatment early after injury may have minimal symptoms with either an innocuous entrance wound or no visible break in the skin. Fusiform swelling resulting from mechanical distention of the tissue by the injectant will usually be apparent. Several hours later, the involved digit or palm may become extremely painful, swollen and pale because of vascular compromise and tissue necrosis [1].

The incidence of high-pressure water injection injury to the mouth or oral cavity appears to be quite rare. A literature search of MEDLINE yielded no reports of highpressure injuries to this region. The only literature detailing an oral water injury was described by Duplechain et al. in 1993 [2]. They reported on a 5-year-old girl who suffered an injury from a much lower pressure bathtub water spout. Here we describe a recent oral injury from a high power pressure washer.

\section{Case report}

A 4-year-old boy presented with his mother to a community emergency department in St. Peters, MO with complaint of a mouth injury. The mother stated that she had been outside cleaning her house with a 2,500 psi power pressure washer. She went inside briefly believing her children were all in the house playing. Upon hearing a scream, she rushed outside to find her 4-year-old son and his sibling playing with the pressure washer. The children, who were used to the squirt nozzle on the garden hose, thought that this item was similar and one child had attempted to give the patient 


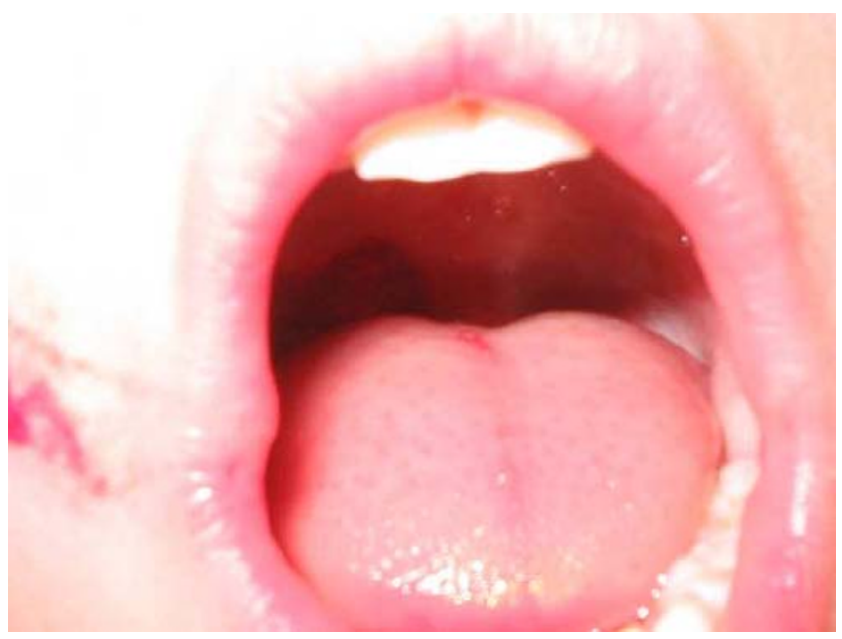

Fig. 1 Macerated right tonsillar pillar

a drink of water from it. The child now had pain and bleeding in the oropharynx. There was no other trauma sustained. The mother of the child immediately brought the patient to the local emergency department.

Examination revealed a crying but easily consolable child sitting on the stretcher with a washcloth in his mouth. Externally, a small abrasion was noted on the cheek and upon inspection of the oropharynx, a macerated right tonsillar pillar was noted (Fig. 1). The child had no difficulty swallowing or breathing. No swelling of the neck was noted. The lungs were clear to auscultation. The rest of the child's exam was unremarkable.

Arrangements were immediately made to transfer the child to St. Louis Children's Hospital (SLCH). Due to concern for possible airway compromise, SLCH's critical care transport team was dispatched. The child was taken by helicopter to SLCH with no problems arising during transport. Upon arrival at SLCH, imaging was obtained. Chest X-ray revealed a pneumomediastinum (Fig. 2). A computed tomography (CT) scan of the neck was also obtained which demonstrated air in the soft tissue of the neck and a small amount of extravasation of contrast material in the right posterior nasopharynx (Fig. 3). Laboratory work was unremarkable except for an elevated white blood cell count of 20,000.

The child was started on Unasyn, dexamethasone and lansoprazole and admitted to the paediatric intensive care unit (PICU) overnight for observation. The next day he was transferred to the ENT floor service. He remained in the hospital for 4 days during which time a carotid Doppler and venous ultrasonography of the neck vessels were obtained.
Fig. 2 Chest X-ray revealing a pneumomediastinum

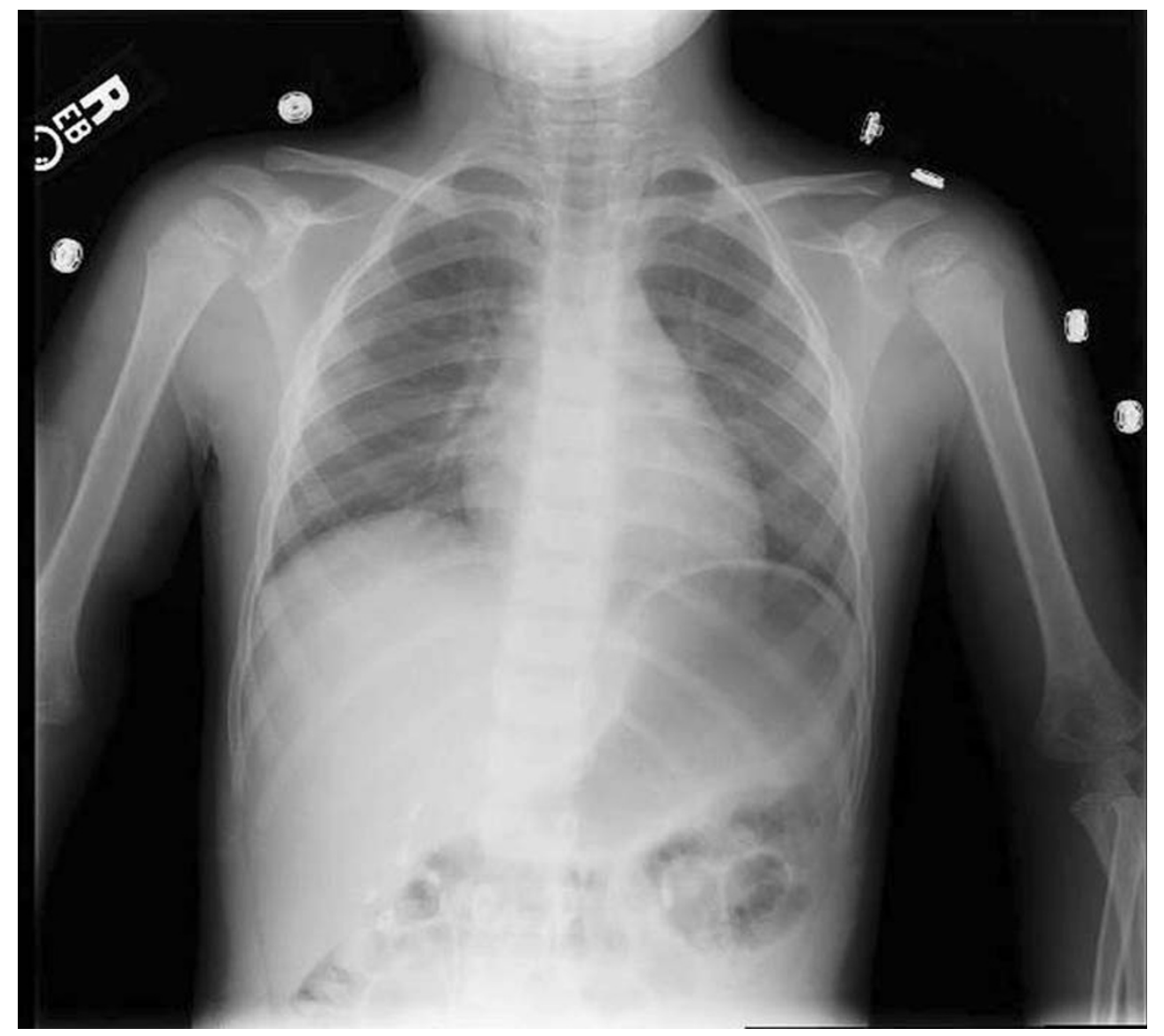


Fig. 3 CT scan of the neck demonstrating air in the soft tissue of the neck and a small amount of extravasation of contrast material in the right posterior nasopharynx

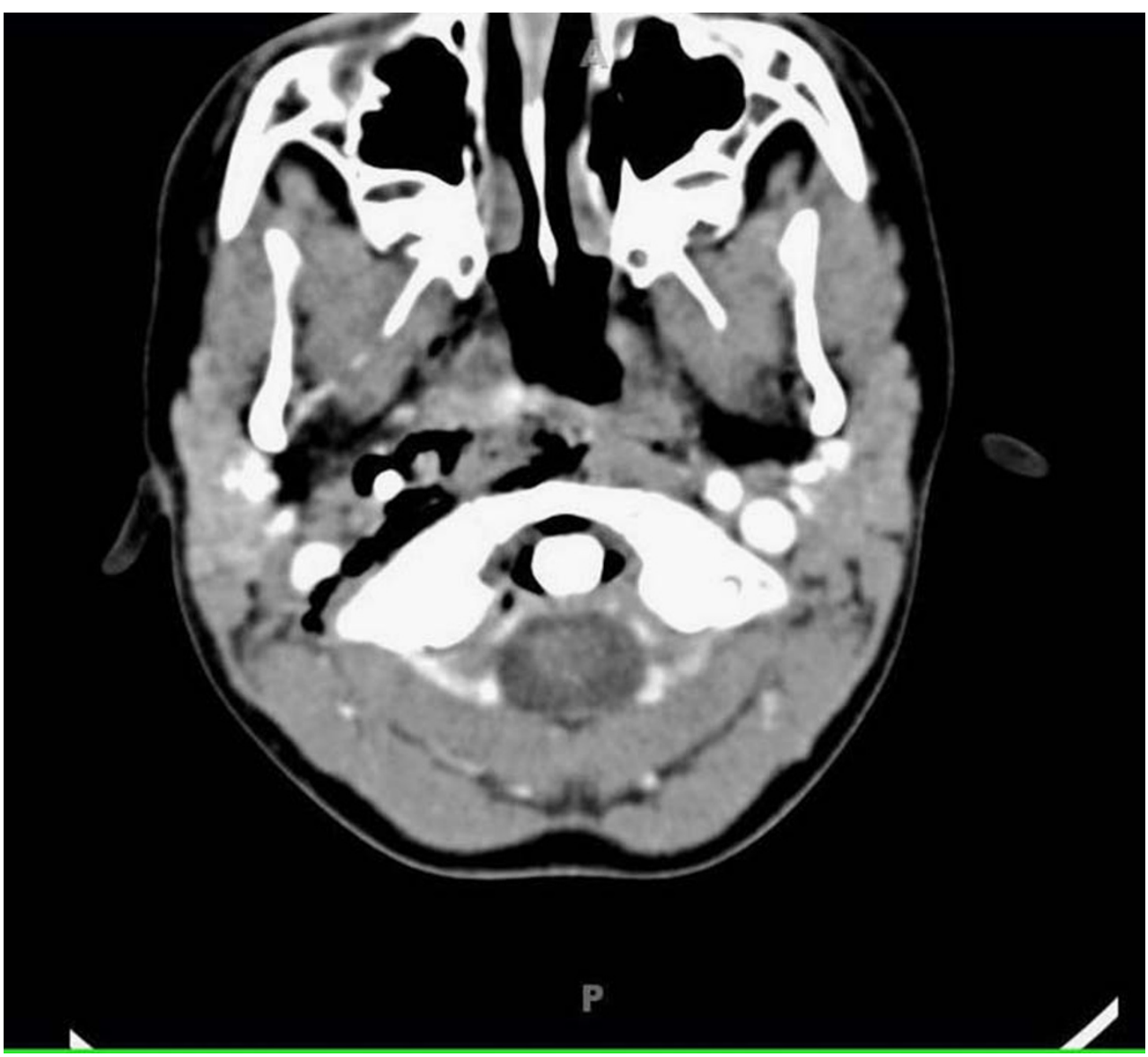

These studies were negative and the patient was then discharged home in good condition.

\section{Discussion}

The above case differs from most high-pressure injection injuries given its location. As with the previous case of water injection injury to the neck, this patient was also managed conservatively with no surgical intervention required. If surgical intervention had been required, the number of significant structures and vessels near the injured area would have made this a complicated undertaking. This patient was injured with a medium duty ranged power washer. These machines range in power from 1,300-2,000 psi for application on cars and boats to heavy duty washer with $2,700-4,000$ psi for stripping paint. Medium duty power washers, like this one, are used for cleaning concrete, siding and decks. The average garden hose emits about $40 \mathrm{psi}$ of pressure [3].

The potential for significant complications in this patient was high. Airway compromise, which may result from either oedema or bleeding, is the most immediate lifethreatening complication. Had there been more extensive damage to the posterior oropharynx, the chance of carotid involvement would have been greatly increased. A significant carotid injury can result in rapid exsanguinations. If arterial or severe venous bleeding had been present, it would have been of primary importance to secure the airway and then confront the source of bleeding. Ideally, endotracheal intubation would be performed first, followed by packing of the oropharynx in order to control bleeding.

Due to potential deterioration of the patient, the critical care transport team at SLCH was used. In cases where airway compromise is a risk, these paediatric patients should be transported by personnel with expertise and appropriate equipment for managing the critically ill child. Barry and Ralston concluded that to reduce unsatisfactory care during transfer of critically ill children, it is necessary to have dedicated and specifically trained transport teams. They supported this with their findings that $75 \%$ of the patients they studied had an adverse clinical event during transport [4].

As with the low-pressure water spout injury described by Duplechain, the intraoral swelling did not increase and the water did not cause any further complications. The patient's airway remained patent throughout his hospital course. Steroids were started to reduce inflammation and potential swelling. A broad-spectrum antibiotic (Unasyn) was started 
to cover any oral flora that may have been introduced into the soft tissues of the neck with the water. The extravasation of contrast material initially seen on the neck CT was likely from an area of localized bleeding that clotted on its own and required no further intervention. Sonography of the carotid and internal jugular veins did not show any injury, supporting the opinion that the bleeding noted above was from smaller vessels.

In summary, high-pressure water injection injuries to the oral cavity appear to be extremely rare. They need to be approached with a high level of awareness of potential complications and possible need for aggressive interven- tion, including early elective intubation in patients who are showing any progression in airway compromise.

\section{References}

1. Marx JA (ed) (2006) Rosen's emergency medicine: concepts and clinical practice, 6th edn. Mosby/Elsevier, Philadelphia

2. Duplechain JK, Espinola T, Miller RH (1993) Water spout injection into the neck. Arch Otolaryngol Head Neck Surg 119:237-238

3. www.pressurewashersdirect.com

4. Barry PW, Ralston C (1994) Adverse events occurring during interhospital transfer of the critically ill. Arch Dis Child 71:8-11 